\title{
Digital approaches for evaluating urban indicators: an application of CityMetrics for analysis of two neighborhoods in Juiz de Fora, Brazil
}

\section{SIGRADI2018 TECHNOPOLITICAS \\ xxii congresso da sociedade iberoamericana de gráfica digital 22th conference of the iberoamerican society of digital graphics 07|08|09|novembro|2018 iau usp | são carlos | sp br}

\author{
Luiza Vallone \\ Federal University of Juiz de Fora | Brazil | luiza.vallone@arquitetura.ufjf.br \\ Frederico Costa \\ Federal University of Juiz de Fora | Brazil | frederico.costa@arquitetura.ufjf.br \\ Lucas Scafutto \\ Federal University of Juiz de Fora | Brazil | lucas.scafutto@arquitetura.ufjf.br \\ Fernando Lima \\ Federal University of Juiz de Fora | Brazil | fernando.lima@arquitetura.ufjf.br
}

\begin{abstract}
This paper describes the application of CityMetrics, a system that articulates computational resources to analyze and optimize the performance of urban configurations. In this context, some indicators (and algorithms) such as Physical and Topological proximity, Spacematrix and Mixed-Use Index were used for analysis, optimization and comparison of two neighborhoods in the city of Juiz de Fora, Brazil. The objective of this research is to verify the possibilities of using computational tools to analyze and propose modifications in neighborhoods and cities, contributing to the decision making in the urban context.
\end{abstract}

Keywords: Urban indicators; CityMetrics; Mixed-Use Index; Spacematrix; Transit Oriented Development.

\section{INTRODUÇÃO}

O presente trabalho é resultado de uma pesquisa de iniciação científica, desenvolvida no âmbito do grupo de pesquisa DOMVS - Laboratório de Investigação em Arquitetura, Urbanismo e Paisagem, vinculado à Faculdade de Arquitetura e Urbanismo da Universidade Federal de Juiz de Fora (FAU-UFJF). Esta investigação, intitulada "Modelagem Algorítmico-Paramétrica e Projetos Urbanos: Métodos Computacionais como suporte à tomada de decisão", se relaciona com outras pesquisas deste mesmo grupo e procura verificar possibilidades de utilização de ferramentas e recursos algorítmicoparamétricos aplicados aos processos de projeto, planejamento e análises urbanas, com vistas a fornecer suporte em processos de tomada de decisão.

O processo migratório de pessoas das zonas rurais para centros urbanizados, conhecido como êxodo rural, teve seu início após a Revolução Industrial e acarretou no aumento da concentração populacional urbana (tanto em termos absolutos, como em termos proporcionais, em relação à população rural). Neste cenário, estima-se que até 2050, 75\% da população mundial estará vivendo em áreas urbanas (ONU, 2013), intensificando a relação entre a qualidade das cidades e o bem-estar da sociedade.

Com esse fenômeno histórico, o aumento da concentração populacional em zonas urbanas promoveu mudanças na estruturação das cidades e um dos paradigmas de planejamento urbano amplamente difundido foi responsável pela consolidação de uma lógica interna insustentável: a malha das cidades, de maneira geral, é organizada com baixa densidade, com suas edificações excessivamente espraiadas e com uma lógica de setorização que não contempla a diversidade de usos, não promove a caminhabilidade e favorece um modo de vida centrado no deslocamento por automóveis.

Neste contexto, o Desenvolvimento Orientado pelo Transporte Sustentável (DOTS) é um modelo de desenvolvimento urbano que preconiza à criação de bairros compactos, caminháveis e de uso misto, organizados em torno de estações de transporte (Lima et al., 2016). Este modelo de organização espacial, que defende a criação de comunidades que não dependem exclusivamente do automóvel para os deslocamentos diários, propõe uma lógica de organização de bairros e cidades mais autônoma e sustentável, baseada em princípios que podem ser objetivamente mensurados, como: (a) acessibilidade ao transporte - 0 posicionamento de estações de transporte próximas a edificação de serviços, comércio e habitação, facilitando os deslocamentos a pé dentro de curtas distâncias para acesso ao transporte coletivo; (b) caminhabilidade capacidade de integração, por meio de distâncias que podem ser percorridas a pé, que uma determinada localidade possui entre diversos serviços urbanos e suas habitações, o que confere mais vitalidade às ruas e ao bairro como um todo; (c) uso misto - diversidade de usos e funções dentro de uma mesma região, o que proporciona maior autonomia para os bairros; (d) densidade - incentivo da alta ocupação populacional dentro dos bairros dotados de infraestrutura, compactando a malha urbana e permitindo assim que 
suporte diferentes modais (Cervero; Kockelman, 1997. Calthorpe; Fulton, 2001. Dittmar; Ohland, 2004. Suzuki et al., 2013).

Portanto, este paper descreve, especificamente, a implementação de CityMetrics: um sistema computacional (algorítmico-paramétrico) desenvolvido por Lima (2017) para fornecer suporte à tomada de decisões em tarefas de planejamento urbano. CityMetrics consiste em uma serie de ferramentas, que articulam métricas e calculam diferentes índices, relacionados aos princípios do DOTS citados anteriormente. Assim, este sistema visa a permitir análises e otimização do desempenho de configurações urbanas, dentro do escopo apresentado.

\section{METODOLOGIA}

Neste cenário, a presente pesquisa consiste na aplicação das ferramentas de CityMetrics em dois casos de estudo na cidade de Juiz de Fora: bairros Cascatinha e Bom Pastor. O objetivo foi 0 de elaborar análises que possibilitem sugestões de melhorias das configurações urbanas estudadas, por meio de ferramentas computacionais e sob a perspectiva dos princípios mensuráveis do DOTS.

Assim, por meio das ferramentas de CityMetrics, foram computacionalmente implementados os seguintes indicadores: Proximidade Física (PF) e Proximidade Topológica (PT) (Lima, 2017); Floor Space Index (FSI), Ground Space Index (GSI) e Network density (N), propostos por Pont \& Haupt (2009) e o índice de uso misto Mixed-Use Index (MXI), proposto por Van den Hoek (2008).

\section{\ALGORITMOS DE PROXIMIDADE FÍSICA (PF) E DE PROXIMIDADE TOPOLÓGICA (PT)}

Os indicadores PF e PT (Lima, 2017) podem ser utilizados tanto no contexto do princípio da acessibilidade aos transportes como no contexto da caminhabilidade. $O$ indicador PF calcula percursos de menores distâncias entre diversas origens e alvos, considerando ainda as inclinações presentes nesses trajetos.

O algoritmo PF considera o posicionamento das origens (determinado lote de um bairro, por exemplo), o desenho das ruas e a localização de um ou mais alvos de interesse para calcular a proximidade física. Dessa forma, quando uma origem (uma residência, por exemplo) está a até $400 \mathrm{~m}$ de distância ( 5 min de caminhada) de um alvo (um determinado serviço urbano, como uma escola), é atribuído a ela um índice de valor igual a 1. À medida que a distância entre origem e alvo se aproxima de 1,6 km (20 minutos a pé), esta pontuação diminui. É atribuído um índice de valor 0 a origens cujas distâncias para o alvo mais próximo sejam maiores ou iguais a 1,6 km (Quadro 01).

Quadro 01: Valores de referência para o indicador de PF. Lima (2017).

Índice Significado

\begin{tabular}{|c|c|}
\hline 1 & Proximidade excelente - menos $400 \mathrm{~m}$ (5min a pé) \\
\hline 0,5 & Boa proximidade $-800 \mathrm{~m}$ (10min a pé) \\
\hline 0 & $\begin{array}{c}\text { Proximidade desconsiderada - mais de } 1600 \mathrm{~m} \text { (20min } \\
\text { a pé) }\end{array}$ \\
\hline
\end{tabular}

O algoritmo PT (Lima 2017), que incorpora conceitos da teoria de Sintaxe Espacial de Hillier \& Hanson (1984), é aplicado para calcular percursos com menores distâncias entre uma determinada origem e um (ou mais) alvo indicado, assim como é capaz de verificar a integração/profundidade dos espaços de uma determinada área. A ferramenta possibilita que se calcule o número de mudanças de direção necessários para se chegar aos alvos indicados partindo de determinada origem, discriminando ainda quais espaços possuem maior integração. Ou seja, através desse indicador, é possível identificar as ruas mais acessíveis e que, consequentemente, impactam mais diretamente na dinâmica das duas áreas estudadas.

\section{ALGORITMO DE INDICADORES SPACEMATRIX}

Esta ferramenta (Lima, 2017) consiste em três indicadores fundamentais propostos por Pont \& Haupt (2009): Intensidade (Floor Space Index - FSI), Cobertura (Ground Space Index - GSI) e Densidade da rede (Network density - N). O FSI aponta a intensidade de construção, e é obtido pela divisão entre a área total construída e a área do local analisado. Já o GSI demonstra a relação entre espaços construídos e não construídos, obtido por meio da razão entre a área ocupada no solo e a área total de análise. $\mathrm{N}$ refere-se à concentração de redes (viárias) em um dado tecido e é encontrado por meio da divisão entre o somatório da extensão das ruas internas com a metade do perímetro das ruas que delimitam uma área, somados a área total do local que está sendo analisado.
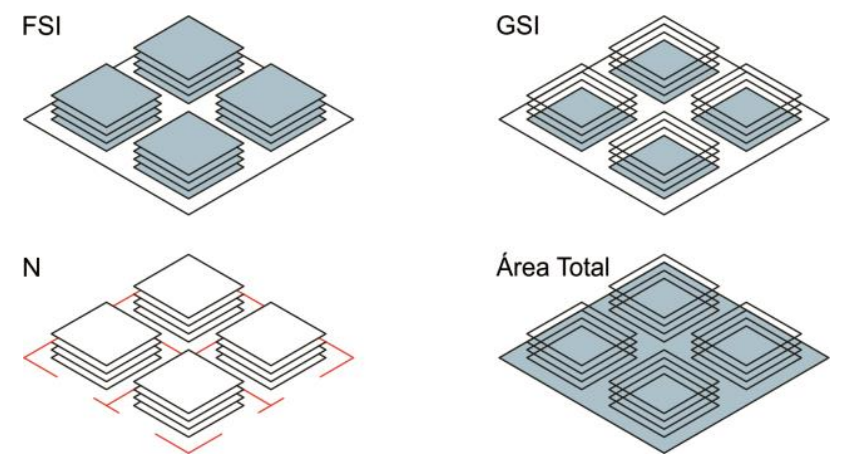

Figura 1: Inputs do Spacematrix para cálculo: área total de pisos (FSI), área de ocupação (GSI), rede de ruas (N) e área total de agregação (no caso desta pesquisa, um bairro). Adaptado de Pont \& Haupt (2009).

\section{ALGORITMO DE USO MISTO (MXI)}

O indicador MXI (Lima 2017), que incorpora o conceito do índice de uso misto (Mixed-use Index - MXI) elaborado por Hoek (2008), é responsável por medir objetivamente a diversidade de uma determinada localidade. Dependendo da abordagem, o índice MXI pode ser, inclusive, configurado como uma das funções objetivo de uma tarefa de otimização, em casos em que a área de estudo tenha uma necessidade de áreas com maior diversidade de usos. A ferramenta calcula a proporção entre o somatório das áreas residenciais e o somatório das áreas não residenciais de uma localidade, realizando uma comparação destas proporções. De acordo com Hoek (2008), quanto mais essa relação se aproxima do equilíbrio dos usos (50/50), maior a diversidade que a área urbana possui. O algoritmo possui grande importância no contexto de CityMetrics, haja visto que 
permite mensurar a diversidade das áreas de estudo antes e depois de potenciais intervenções sugeridas ao longo do processo de análise.

Quadro 02: Valores de referência para o MXI. Adaptado de Van den Hoek (2008).

\begin{tabular}{|c|c|c|c|}
\hline Valor MXI & $\mathbf{0}$ & $\mathbf{5 0}$ & $\mathbf{1 0 0}$ \\
\hline Significado & $\begin{array}{c}100 \% \text { uso não } \\
\text { residencial }\end{array}$ & $\begin{array}{c}\text { Uso } \\
\text { equilibrado } \\
\text { em } 50 / 50\end{array}$ & $\begin{array}{c}100 \% \text { uso } \\
\text { residencial }\end{array}$ \\
\hline $\begin{array}{c}\text { Tipo de } \\
\text { região }\end{array}$ & Monofuncional & Uso misto & Monofuncional \\
\hline Exemplos & $\begin{array}{c}\text { Complexo } \\
\text { industrial ou } \\
\text { comercial / } \\
\text { serviços }\end{array}$ & $\begin{array}{c}\text { Centro e } \\
\text { zonas } \\
\text { próximas ao } \\
\text { centro da } \\
\text { cidade }\end{array}$ & $\begin{array}{c}\text { Áreas } \\
\text { periféricas / } \\
\text { subúrbio }\end{array}$ \\
\hline
\end{tabular}

\section{EXPERIMENTOS}

A escolha dos bairros para a elaboração dos experimentos se deu pelos seguintes aspectos: Bairros da cidade de Juiz de Fora, com possibilidade de adensamento e verticalização, próximos a região central, dotados de vias articuladoras importantes e com carência de uma unidade de transporte consolidada. Ambas as regiões são tipicamente residenciais, com baixo uso misto. O bairro Bom Pastor apresenta menor quantidade de edifícios do tipo multifamiliar e mais residências unifamiliares em relação ao bairro Cascatinha.

Para serem analisados através do sistema CityMetrics, as áreas de estudo, bairros Cascatinha (A) e Bom Pastor (B), passaram por processos de levantamento de dados e de preparação de arquivos dentro de softwares específicos, na seguinte ordem:

No software AutoCAD®, os procedimentos se desenvolveram a partir (1) da escolha das áreas de recorte, estabelecida pelo núcleo central, principais vias do bairro e arredores imediatos que poderiam impactar nas análises (2) Limpeza e padronização do arquivo .DWG e (3) transformação das linhas de perímetro de cada terreno, das linhas de perímetro de cada edificação e dos eixos das vias em polylines.

Em seguida, foi feito um levantamento in loco, com o objetivo de elaborar (4) uma análise dos gabaritos, indexando as alturas das edificações, (5) uma notação de quantos eram os pavimentos residenciais e não residenciais presentes em cada uma das edificações.

Com auxílio do programa Excel®, (6) foi criada uma tabela com o número de pavimentos de cada edificação e, em seguida, (7) uma quantificação de pavimentos residenciais e pavimentos não residenciais;

Depois de todos esses processos, os dados foram introduzidos no software Rhinoceros $\AA$, para aplicar 0 sistema CityMetrics, com os recursos do plug-in de modelagem algorítmica Grasshopper®. As etapas se desenvolveram na seguinte ordem: (1) definir as curvas das edificações, dos terrenos e das vias separadamente; (2) definir os centros geométricos numerados das edificações através da ferramenta 'area'; (3) importar a lista com os dados de pavimentos das respectivas edificações; (4) importar curva delimitando o recorte analisado e as curvas do eixos das ruas internas e externas, (5) definir os centroides referentes a cada serviço existente na área e agrupa-los; (6) criar a superfície referente a topografia existente e projetar as curvas das vias, edificações e centroides; (7) definir os "lotes potenciais" (lotes vazios ou com edificações com menos de 2 pavimentos); (8) aplicar os algoritmos do CityMetrics - MXI, Spacematrix, PF e PT, (9) aplicar métodos de otimização para propor novos serviços e, consequentemente, novos índices MXI, PF e PT; (10) designar novas edificações ou usos aos lotes vazios, e; (11) aplicar CityMetrics para a nova configuração do recorte analisado.

Após essa análise, foram utilizadas, dentro do plug-in Grasshopper®, tarefas de otimização para sugerir a inserção de novos serviços nos dois bairros, buscando atingir configurações espaciais e arranjos que oferecessem um melhor desempenho para os bairros, sob a perspectiva dos indicadores abordados nesta pesquisa.

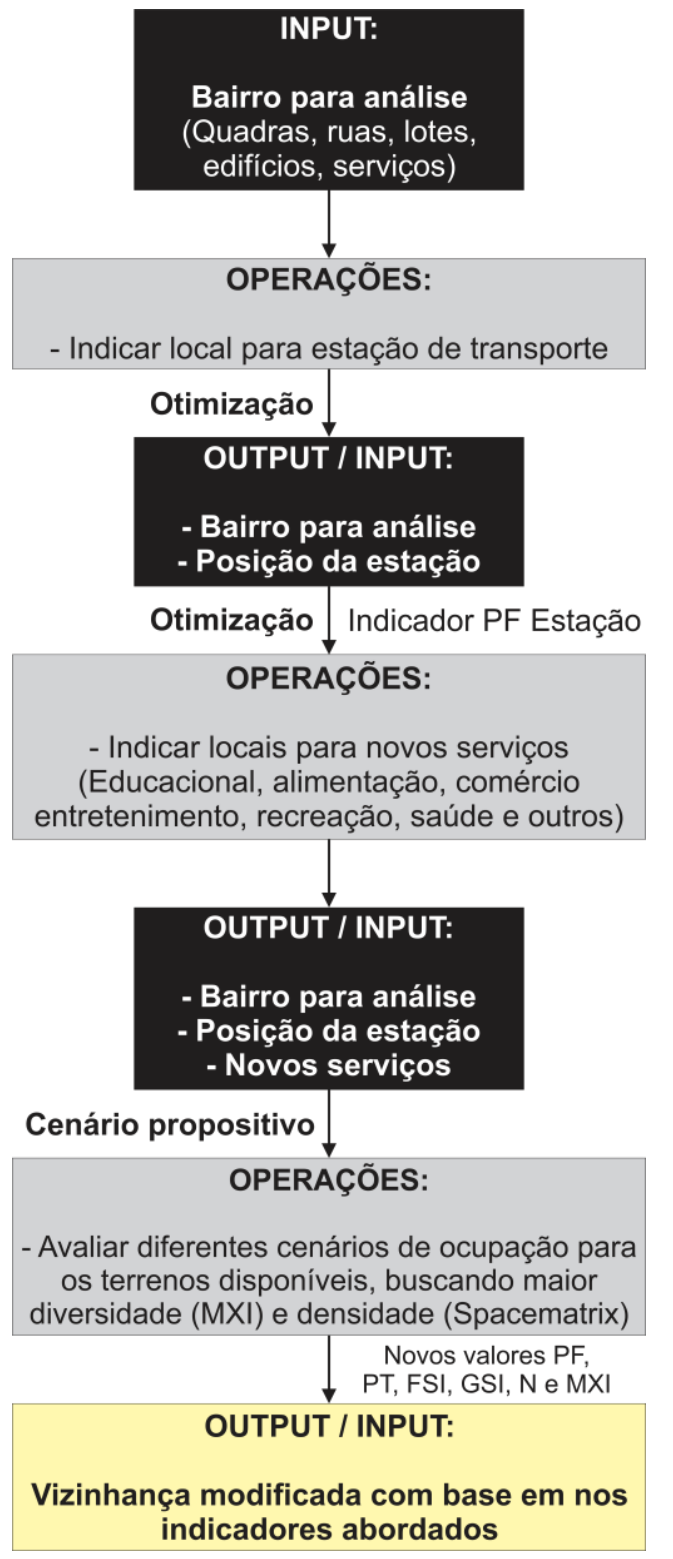

Figura 2: Esquema do processo metodológico adotado para desenvolver os experimentos A e B. Os autores (2018). 


\section{RESULTADOS}

Como resultado dos experimentos desenvolvidos por essa pesquisa, foi possível verificar que ferramentas computacionais como a de CityMetrics devem ser entendidas como instrumentos de auxílio, atuando em ocasiões que seja possível inserir parâmetros mensuráveis (métricas) nos processos de planejamento urbano, tanto na análise quanto nas otimizações.

Em relação aos experimentos, foram computacionalmente obtidos os indicadores PF, PT, FSI, GSI, N e MXI dos dois bairros estudados, o que possibilitou uma análise da situação atual destas localidades sob o ponto de vista da proximidade física e da proximidade topológica (acessibilidade ao transporte e caminhabilidade), da densidade urbana (compacidade) e da diversidade de usos.

Através dos estudos de caso também foi possível verificar a utilidade do sistema adotado. Através da utilização de CityMetrics, foram obtidos dados objetivos que confirmaram o que a equipe de pesquisa já esperava encontrar após uma observação empírica das duas áreas de estudo: tratam-se de dois bairros com pouca diversidade de usos (baixo MXI), baixa densidade urbana, caminhabilidade restrita e dependência de meios de transporte motorizados para acessar uma variedade maior de serviços.

$\mathrm{L}$

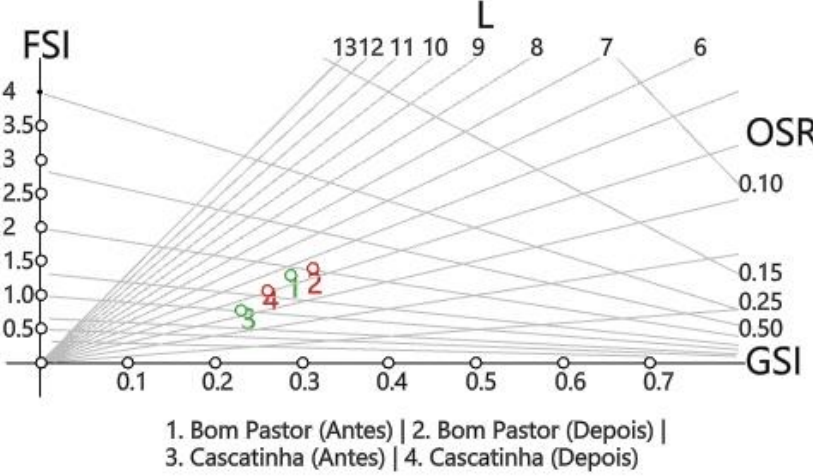

Figura 3: Gráfico representativo dos indicadores Spacematrix para os bairros Cascatinha e Bom pastor, antes e depois das análises e otimizações. Adaptado de Pont \& Haupt (2009)

\section{A) BAIRRO CASCATINHA}

O experimento no bairro Cascatinha, realizado por Lima (2017), adotou o plug-in Octopus $\AA^{\circledR}$ para execução das tarefas de otimização, considerando a lógica da otimização multi-objetivo, que permite abordar diversas funções objetivas simultaneamente. A otimização multiobjetivo apresenta uma solução na forma do gráfico da fronteira de Pareto, onde todas as soluções apresentadas em determinada fronteira são consideradas igualmente satisfatórias, dentro da perspectiva de todos os critérios adotados para análise.

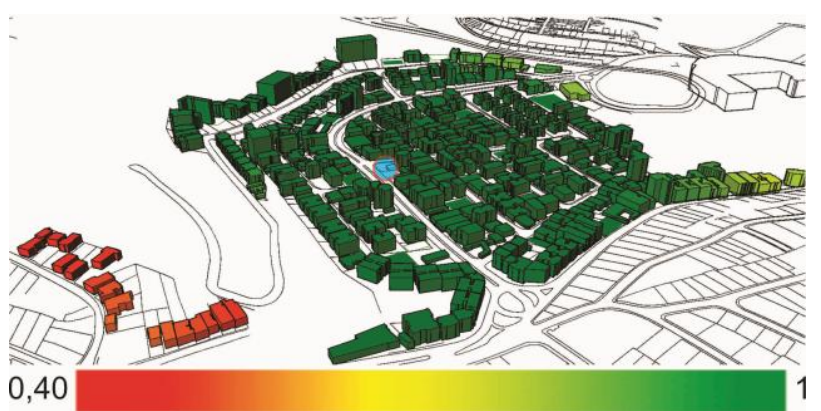

Figura 4: Proximidade Física entre os edifícios do bairro Cascatinha e a estação (em azul), cuja localização foi indicada por meio de otimização. Cada edificação se encontra preenchida por uma cor que corresponde ao seu índice de PF para a estação. Lima (2017).

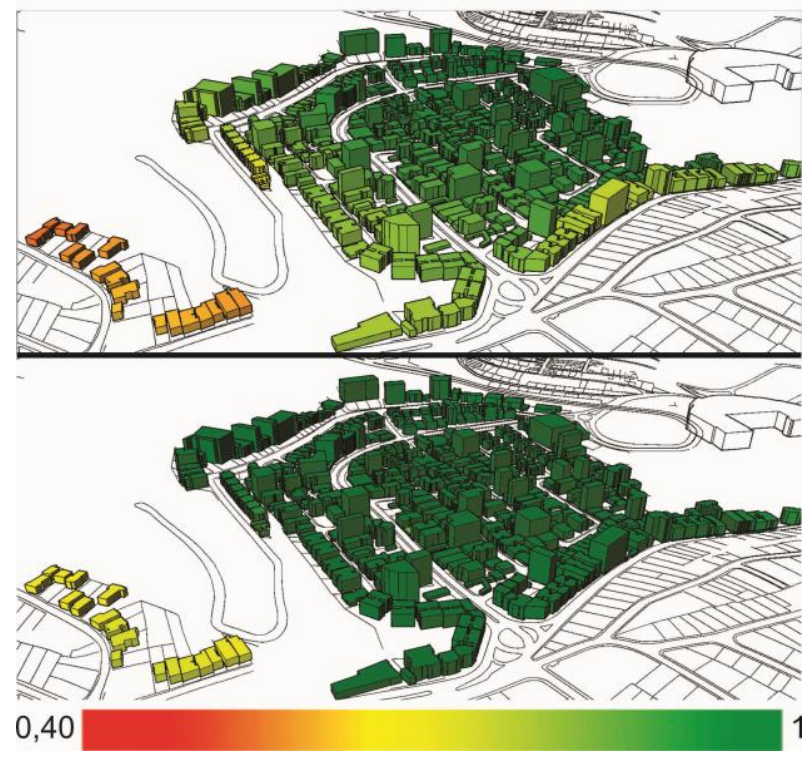

Figura 5: Proximidade Física Global entre os edifícios e os serviços no bairro Cascatinha, antes e depois das otimizações. Lima (2017)

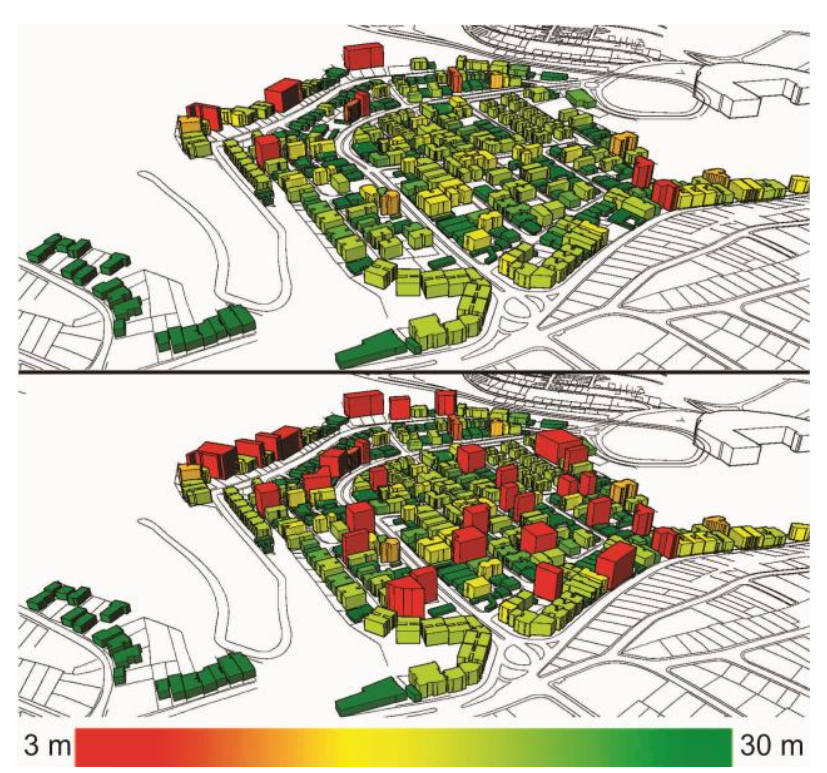

Figura 6: Estudo de alturas e da densidade após implementação de CityMetrics. As otimizações propostas para o bairro proporcionaram indicadores de densidade mais elevados. Lima (2017). 
Tabela 01: Apresentação dos resultados obtidos no experimento A, alcançados a partir das análises realizadas no Bairro Cascatinha, indexando a área quanto a diversidade (MXI) e densidade urbana (Spacematrix). Lima (2017).

\begin{tabular}{|c|c|c|c|c|c|}
\hline \multicolumn{7}{|c|}{ Bairro Cascatinha } \\
\hline \multirow{2}{*}{ Diversidade e Densidade (antes/depois) } \\
\cline { 2 - 6 } \multicolumn{2}{|c|}{ Residencial } & \multicolumn{2}{|c|}{$\tilde{\text { N}}$-Residencial } \\
\cline { 2 - 6 } & 0,84 & $\underline{0,58}$ & 0,16 & $\underline{0,42}$ \\
\hline \multicolumn{7}{|c|}{ Spacematrix } \\
\hline \multicolumn{2}{|c|}{ GSI } & \multicolumn{2}{c|}{$\mathrm{N}$} \\
\hline 0,77 & $\underline{1,12}$ & 0,23 & $\underline{0,26}$ & 0,21 & $\underline{0,21}$ \\
\hline
\end{tabular}

Tabela 02: Apresentação de informações gerais e de resultados obtidos para 0 experimento $A$, alcançados a partir da análise realizada no Bairro Cascatinha, com números comparativos e os dados relativos aos indicadores PF e PT. Lima (2017).

\begin{tabular}{|c|c|c|c|c|c|c|}
\hline \multicolumn{7}{|c|}{$\begin{array}{l}\text { Bairro Cascatinha } \\
\text { ações Gerais (antes/g }\end{array}$} \\
\hline \multicolumn{3}{|l|}{ Área toral do bairro } & \multicolumn{2}{|c|}{42,48 ha } & \multicolumn{2}{|c|}{42,48 ha } \\
\hline \multicolumn{3}{|l|}{ Número total de quadras } & \multicolumn{2}{|c|}{17} & \multicolumn{2}{|c|}{17} \\
\hline \multicolumn{3}{|l|}{ Número toral de lotes } & \multicolumn{2}{|c|}{426} & \multicolumn{2}{|c|}{426} \\
\hline \multicolumn{3}{|l|}{ Número total de edifícios } & \multicolumn{2}{|c|}{397} & \multicolumn{2}{|c|}{$\underline{423}$} \\
\hline \multicolumn{7}{|c|}{ Acessibilidade ao Transporte } \\
\hline \multicolumn{7}{|c|}{ Distâncias entre os lotes e a estação } \\
\hline Menor & \multicolumn{3}{|c|}{ Média } & \multicolumn{3}{|c|}{ Maior } \\
\hline $20 \mathrm{~m}$ & \multicolumn{3}{|c|}{$335 \mathrm{~m}$} & \multicolumn{3}{|c|}{$1053 \mathrm{~m}$} \\
\hline \multicolumn{7}{|c|}{ Índices de Proximidade Física (IPF) p/ estação } \\
\hline Menor & \multicolumn{3}{|c|}{ Média } & \multicolumn{3}{|c|}{ Maior } \\
\hline 0,4 & \multicolumn{3}{|c|}{0,95} & \multicolumn{3}{|c|}{1} \\
\hline \multicolumn{7}{|c|}{ Caminhabilidade (antes/depois) } \\
\hline \multicolumn{7}{|c|}{ Indice de Proximidade Física (IPF) p/ serviços (P } \\
\hline Categoria & \multicolumn{2}{|c|}{ Menor } & & & & \\
\hline Educacional & 0,73 & 0,74 & 0,97 & 0,99 & 1 & 1 \\
\hline Alimentação & 0,68 & 0,68 & 0,98 & 0,99 & 1 & 1 \\
\hline Comércio & 0,62 & 0,60 & 0,98 & 0,98 & 1 & 1 \\
\hline Entretenimento & 0 & 0,42 & 0,53 & 0,94 & 0,96 & 1 \\
\hline Recreação & 0,59 & 0,75 & 0,89 & 0,98 & 1 & 1 \\
\hline Saúde & 0 & $\underline{0,58}$ & 0,80 & 0,96 & 1 & 1 \\
\hline Outros & 0,56 & $\underline{0,58}$ & 0,97 & 0,99 & 1 & $\underline{1}$ \\
\hline Índices de PrC & kimida & Física & F) $p / s$ & viços ( & lobais) & \\
\hline Menor & & Média & & & Maio & \\
\hline 0,72 & & & 0,98 & & 99 & 1 \\
\hline Índices de Prox & nidade & opológ & $-\mathrm{Sen}$ & OS (IPT & (Parcia & \\
\hline Categoria & & & & & & \\
\hline Educacional & 0 & $\underline{0}$ & 1,57 & 1,43 & 5 & $\underline{5}$ \\
\hline Alimentação & 0 & $\underline{0}$ & 1,41 & 1,08 & 7 & 5 \\
\hline Comércio & 0 & $\underline{0}$ & 1,68 & 1,35 & 7 & $\underline{5}$ \\
\hline Entretenimento & 0 & $\underline{0}$ & 5,49 & 2,35 & 10 & $\underline{7}$ \\
\hline Recreação & 0 & 0 & 2,51 & 1,97 & 8 & 7 \\
\hline Saúde & 0 & $\underline{0}$ & 1,84 & 1,51 & 6 & $\underline{6}$ \\
\hline Outros & 0 & $\underline{0}$ & 1,70 & 1,41 & 6 & 6 \\
\hline Índices de Prox & nidade & opológ & -Sen & OS (IPT & (Globa & \\
\hline Menor & & Média & & & Maio & \\
\hline 0,57 & & & 1,59 & & 7 & 5,89 \\
\hline
\end{tabular}

\section{B) BAIRRO BOM PASTOR}

No experimento realizado no bairro Bom Pastor, as tarefas de otimização foram realizadas no software Rhinoceros $\AA$, através do plug-in Galapagos $\AA$, que considera apenas uma função objetiva para realizar os processos de otimização. Este tipo de otimização fornece uma lista das soluções ótimas, em ordem crescente ou decrescente, de acordo com condições preestabelecidas.

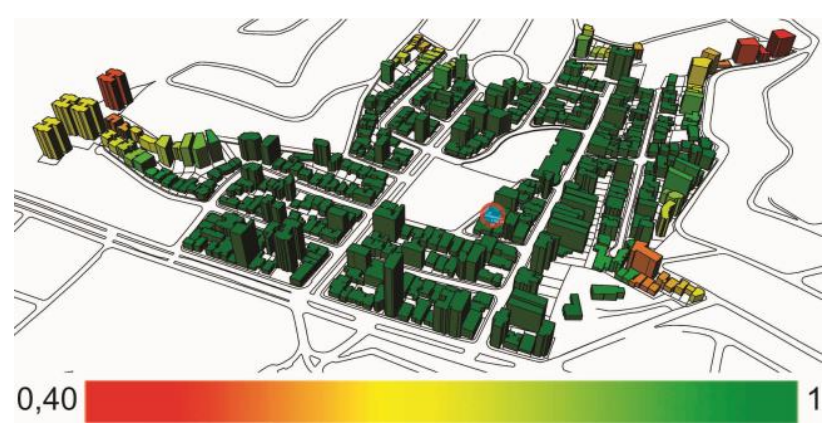

Figura 7: Proximidade Física entre os edifícios do bairro Bom Pastor e a estação (em azul), cuja localização foi indicada por meio de otimizações. As edificações se encontram preenchidas por uma cor que corresponde ao seu índice de PF para a estação. Os autores (2018).

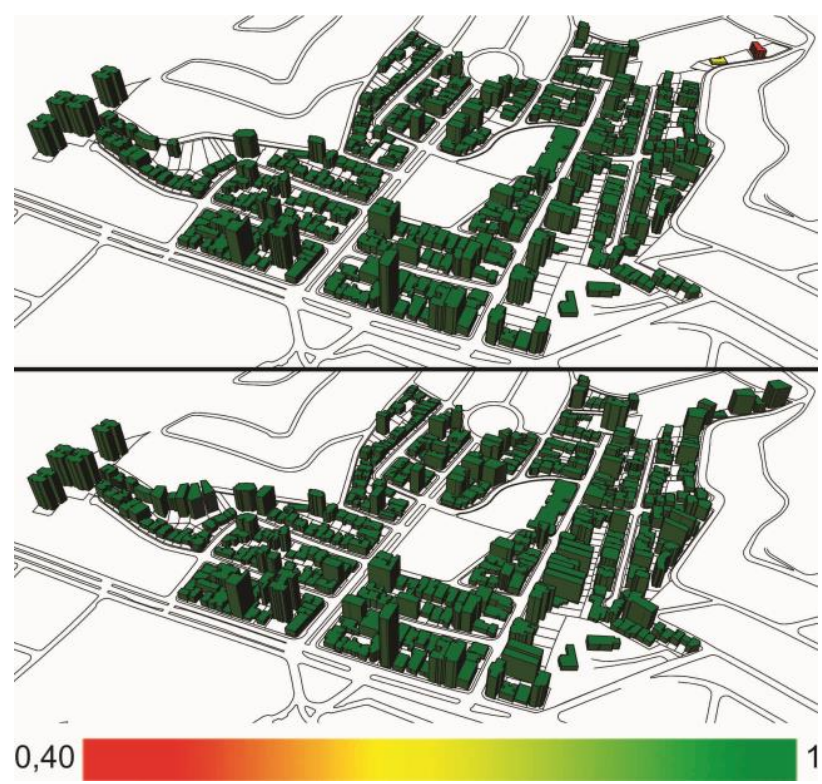

Figura 8: Proximidade Física Global entre os edifícios e os serviços no bairro Bom Pastor, antes e depois das otimizações. Os autores (2018).

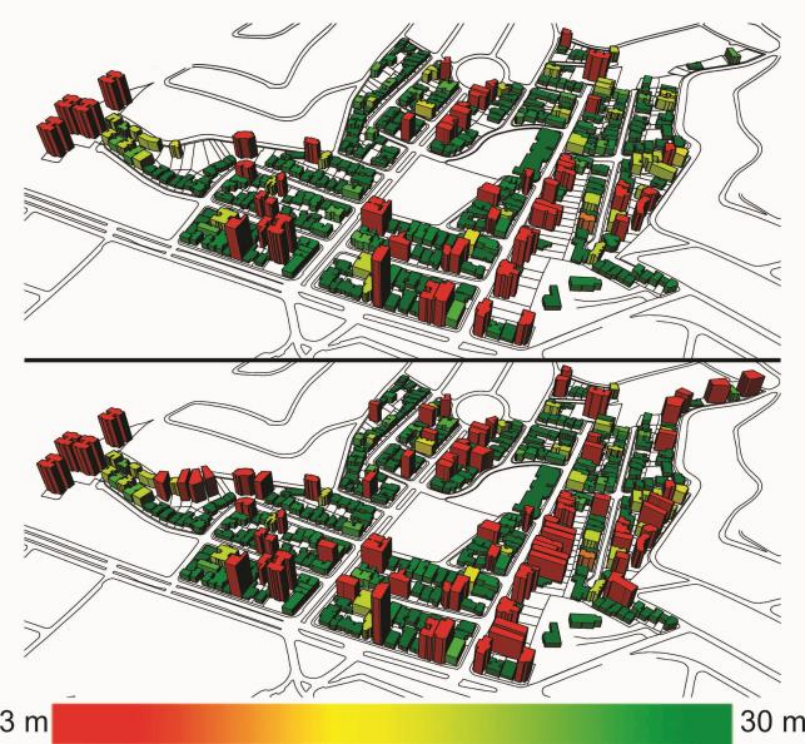

Figura 9: Estudo de alturas e da densidade dos edifícios do bairro Bom Pastor após implementação de CityMetrics. Otimizações propostas elevaram os indicadores de densidade e MXI. Os autores (2018). 
Tabela 03: Apresentação dos resultados obtidos no experimento $\mathrm{B}$, alcançados a partir das análises realizadas no Bairro Bom Pastor, indexando a área quanto a diversidade (MXI) e densidade urbana (Spacematrix).

\begin{tabular}{|c|c|c|c|c|c|}
\hline \multicolumn{6}{|c|}{$\begin{array}{l}\text { Bairro Bom Pastor } \\
\text { dade e Densidade (antes/depois) }\end{array}$} \\
\hline \multirow{2}{*}{\multicolumn{2}{|c|}{ Uso Misto (MXI) }} & \multicolumn{2}{|c|}{ Residencial } & \multicolumn{2}{|c|}{$\tilde{\tilde{N}}$-Residencial } \\
\hline & & 0,944 & 0,758 & 0,005 & 0,241 \\
\hline \multicolumn{6}{|c|}{ Spacematrix } \\
\hline \multicolumn{2}{|c|}{$\mathrm{FSI}$} & \multicolumn{2}{|c|}{ GSI } & \multicolumn{2}{|c|}{$\mathrm{N}$} \\
\hline 1,128 & 1,392 & 0,286 & 0,312 & 0,015 & 0,015 \\
\hline
\end{tabular}

Tabela 04: Apresentação de informações gerais e de resultados obtidos para o experimento $\mathrm{B}$, alcançados a partir da análise realizada no Bairro Bom Pastor, com números comparativos e os dados relativos aos indicadores PF e PT.

\begin{tabular}{|c|c|c|c|c|c|c|}
\hline \multicolumn{7}{|c|}{$\begin{array}{l}\text { Bairro Bom Pastor } \\
\text { ções Gerais (antes/depois) }\end{array}$} \\
\hline \multicolumn{3}{|l|}{ Área toral do bairro } & \multicolumn{2}{|c|}{$32,39 \mathrm{ha}$} & \multicolumn{2}{|c|}{ 32,39ha } \\
\hline \multicolumn{3}{|c|}{ Número total de quadras } & \multicolumn{2}{|c|}{14} & \multicolumn{2}{|c|}{$\underline{14}$} \\
\hline \multicolumn{3}{|l|}{ Número toral de lotes } & \multicolumn{2}{|c|}{414} & \multicolumn{2}{|c|}{414} \\
\hline \multicolumn{3}{|c|}{ Número total de edifícios } & \multicolumn{2}{|c|}{384} & \multicolumn{2}{|c|}{$\overline{414}$} \\
\hline \multicolumn{7}{|c|}{ Acessibilidade ao Transporte } \\
\hline \multicolumn{7}{|c|}{ Distâncias entre os lotes e a estação } \\
\hline Menor & \multicolumn{3}{|c|}{ Média } & \multicolumn{3}{|c|}{ Maior } \\
\hline $15 \mathrm{~m}$ & \multicolumn{3}{|c|}{$363 \mathrm{~m}$} & \multicolumn{3}{|c|}{$662 m$} \\
\hline \multicolumn{7}{|c|}{ Índices de Proximidade Física (IPF) p/ estação } \\
\hline Menor & \multicolumn{3}{|c|}{ Média } & \multicolumn{3}{|c|}{ Maior } \\
\hline 0,73 & \multicolumn{3}{|c|}{0,961} & \multicolumn{3}{|c|}{1} \\
\hline \multicolumn{7}{|c|}{ Caminhabilidade (antes/depois) } \\
\hline \multicolumn{7}{|c|}{ Indice de Proximidade Física (IPF) p/ serviços } \\
\hline Categoria & $\mathrm{Me}$ & nor & & & & \\
\hline Educacional & 0,539 & $\underline{0,7533}$ & 0,925 & $\underline{0,978}$ & 1 & $\underline{1}$ \\
\hline Alimentação & 0,776 & $\underline{0,856}$ & 0,991 & $\underline{\underline{0,998}}$ & 1 & $\underline{\underline{1}}$ \\
\hline Comércio & 0,624 & 0,828 & 0,941 & $\underline{0,993}$ & 1 & $\underline{1}$ \\
\hline Entretenimento & 0 & 0,719 & 0 & 0,957 & 0 & $\underline{1}$ \\
\hline Recreação & 0,833 & 0,849 & 0,988 & 0,994 & 1 & $\underline{1}$ \\
\hline Saúde & 0,704 & $\underline{0,704}$ & 0,986 & $\underline{0,994}$ & 1 & $\underline{1}$ \\
\hline Outros & 0,702 & 0,878 & 0,99 & 0,997 & 1 & 1 \\
\hline Índices de F & kimidad & Física (I & F) $p / s e$ & viços (C & lobais) & \\
\hline Menor & & Média & & & Maior & \\
\hline 0,902 & & 99 & $\underline{1}$ & & 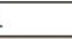 & $\underline{1}$ \\
\hline Índices de Pro & dade & opológic & a-Servi & os (IPT) & Parciai & \\
\hline Categoria & & nor & & & & \\
\hline Educacional & 0 & $\underline{0}$ & 4,26 & 2,739 & 10 & $\underline{7}$ \\
\hline Alimentação & 0 & $\underline{0}$ & 1,978 & 1,826 & 6 & $\underline{6}$ \\
\hline Comércio & 0 & $\underline{0}$ & 3,326 & 2,108 & 7 & $\underline{5}$ \\
\hline Entretenimento & 0 & $\underline{0}$ & 0 & $\underline{3,934}$ & 0 & $\underline{8}$ \\
\hline Recreação & 0 & $\underline{0}$ & 2,521 & $\underline{2,195}$ & 5 & $\underline{5}$ \\
\hline Saúde & 0 & $\underline{0}$ & 2,586 & 2,065 & 7 & $\underline{5}$ \\
\hline Outros & 0 & $\underline{0}$ & 1,934 & $\underline{1,521}$ & 7 & $\underline{4}$ \\
\hline Índices de Pro & idade & opológic & a-Servi & OS (IPT) & Globai & \\
\hline Menor & & Média & & & Maior & \\
\hline$\underline{0}$ & & 13 & $\underline{\underline{1}}$ & & 5 & $\underline{4}$ \\
\hline
\end{tabular}

Com base nas análises que foram articuladas, o sistema também viabilizou a visualização, em ambiente digital, de diferentes cenários propositivos para intervenção nas áreas de estudo, o que levou a equipe propor otimizações que atuassem diretamente em cima dos indicadores adotados para avaliação nos dois experimentos. O sistema CityMetrics e a metodologia utilizada se mostraram eficientes, haja vista que após os processos de otimização foi possível melhorar os índices (PF, PT, FSI, GSI, N e MXI) que foram abordados por essa pesquisa em ambos os casos de estudo.

\section{DISCUSSÃO}

Através dos dados obtidos nos resultados, foi possível identificar que ambos os bairros possuem baixa densidade urbana e que os usos são pouco diversificados nas duas regiões escolhidas para os experimentos. Assim, ficou evidente que tal fato se deve, entre outros aspectos, ao histórico de ocupação dos bairros e, principalmente, ao modelo de desenvolvimento urbano permitido pela legislação do município de Juiz de Fora. As possibilidades de ocupação nas duas áreas estudadas, ainda que em cenários de proposição extrema, não permite que os bairros possuam uma densidade adequada, sob o ponto de vista dos Indicadores Spacematrix. No que tange aos usos das regiões estudadas, ambas não possuem um bom equilíbrio sob o ponto de vista do índice MXI, o que acaba influenciando nos índices PF e PT, pois é necessário um deslocamento maior para acessar serviços diversos.

As ferramentas computacionais expuseram características das duas configurações urbanas estudadas (Experimentos A e B), inclusive aquelas que se relacionam diretamente com os códigos de postura e outros dispositivos legais das cidades, permitindo até que se verifique necessidades de atualizações nos mesmos. Destaca-se que o emprego de ferramentas algorítmicoparamétricas em tarefas de análise / otimização em contextos urbanos ainda constitui um campo em desenvolvimento. Neste cenário, esta pesquisa pretende contribuir para uma utilização mais efetiva dos recursos computacionais como ferramenta de estudo e de suporte à tomada de decisões em processos de planejamento de bairros e cidades.

\section{AGRADECIMENTOS}

Os autores gostariam de agradecer ao Conselho Nacional de Desenvolvimento Científico e Tecnológico (CNPq), a Pró-reitoria de Pós-graduação e Pesquisa da Universidade Federal de Juiz de Fora (PROPP UFJF) e ao Programa de Pós-graduação em Ambiente Construído (PROAC).

\section{REFERÊNCIAS}

Calthorpe, P.; Fulton, W. (2001). The regional city: planning for the end of sprawl. Washington: Island Press.

Cervero, R.; Kockelman, K. (1997). Travel demand and the 3Ds: density, diversity, and design. Transportation Research Part D: Transport and Environment, 2, 199-219.

Dittmar, H.; Ohland, G. (2004). The new transit town: Best practices in transit-oriented development. Washington: Island Press.

Hillier, B.; Hanson, J. The social logic of space. Cambridge: Cambridge University Press, 1984.

Lima, F. Métricas Urbanas: Sistema (para)métrico para análise e otimização de configurações urbanas de acordo com métricas de avaliação de desempenho. Rio de Janeiro, 2017. Tese (Doutorado em Urbanismo) Faculdade de Arquitetura e Urbanismo, Universidade Federal do Rio de Janeiro, Rio de Janeiro, 2017.

Lima, F.; Kos, J. R.; Montenegro, N.; "Otimização multi-objetivo e Desenvolvimento Orientado pelo Transporte: algoritmos evolutivos em estratégias de planejamento urbano", p. 601608. In: XX Congreso de la Sociedad Iberoamericana de Gráfica Digital [=Blucher Design Proceedings, v.3 n.1]. São Paulo: Blucher, 2016. 
Organização das Nações Unidas. Retrieved from: https://nacoesunidas.org/onu-mais-de-70-da-populacaomundial-vivera-em-cidades-ate-2050/

Pont, M. B.; Haupt, P. (2009). Spacematrix: Space, Density and Urban Form. Rotterdam: NAI Publishers, 2009.
Suzuki, H.; Cervero, R.; luchi, K. (2013). Transforming cities with transit: Transit and land-use integration for sustainable urban development. Washington: Word Bank.

Van den Hoek, J. W. (2008) The MXI (Mixed-use Index) as Tool for Urban Planning and Analysis. (Paper 03) In Corporations and Cities: Envisioning Corporate Real Estate in the Urban Future. Available 26 May 2008. Delft, Holanda: TU Delft, 2008. Disponível em: www.corporationsandcities.org 